\title{
Evaluation and selection of tandem repeat loci for a Brucella MLVA
} typing assay

Philippe Le Flèche ${ }^{1,2}$, Isabelle Jacques ${ }^{3,4}$, Maggy Grayon ${ }^{3}$, Sascha Al Dahouk ${ }^{5}$, Patrick Bouchon ${ }^{1,2}$, France Denoeud ${ }^{2}$, Karsten Nöckler ${ }^{6}$, Heinrich Neubauer ${ }^{5}$, Laurence A Guilloteau ${ }^{3}$ and Gilles Vergnaud*1,2

Address: ${ }^{1}$ Centre d'Etudes du Bouchet BP3, 91710 Vert le Petit, France, ${ }^{2}$ GPMS, Bât. 400, Institut de Génétique et Microbiologie, Université Paris Sud, 91405 Orsay cedex, France, ${ }^{3}$ UR918 - Laboratoire de Pathologie Infectieuse et Immunologie, Institut National de la Recherche Agronomique, 37380 Nouzilly, France, ${ }^{4}$ Institut Universitaire de Technologie, 29 rue du Pont Volant, 37082 Tours Cedex 2, France, ${ }^{5}$ Bundeswehr Institute of Microbiology, Dept. of Bacteriology, Neuherbergstr. 11, D-80937 Munich, Germany and ${ }^{6}$ Federal Institute for Risk Assessment, BfR, Diedersdorfer Weg 1, D-12277 Berlin, Germany

Email: Philippe Le Flèche - lefleche@igmors.u-psud.fr; Isabelle Jacques - Isabelle.Jacques@tours.inra.fr; Maggy Grayon - grayon@tours.inra.fr; Sascha Al Dahouk - saschaaldahouk@bundeswehr.org; Patrick Bouchon - Patrick.Bouchon@polytechnique.org;

France Denoeud - France.Denoeud@igmors.u-psud.fr; Karsten Nöckler - k.noeckler@bfr.bund.de;

Heinrich Neubauer - HeinrichNeubauer@bundeswehr.org; Laurence A Guilloteau - guillote@tours.inra.fr;

Gilles Vergnaud* - Gilles.Vergnaud@igmors.u-psud.fr

* Corresponding author

Published: 09 February 2006

BMC Microbiology 2006, 6:9 doi:| 0.| | 86/| 47| |-2|80-6-9
Received: 10 October 2005

Accepted: 09 February 2006

This article is available from: http://www.biomedcentral.com/I47I-2/80/6/9

(c) 2006 Le Flèche et al; licensee BioMed Central Ltd.

This is an Open Access article distributed under the terms of the Creative Commons Attribution License (http://creativecommons.org/licenses/by/2.0), which permits unrestricted use, distribution, and reproduction in any medium, provided the original work is properly cited.

\begin{abstract}
Background: The classification of Brucella into species and biovars relies on phenotypic characteristics and sometimes raises difficulties in the interpretation of the results due to an absence of standardization of the typing reagents. In addition, the resolution of this biotyping is moderate and requires the manipulation of the living agent. More efficient DNA-based methods are needed, and this work explores the suitability of multiple locus variable number tandem repeats analysis (MLVA) for both typing and species identification.

Results: Eighty tandem repeat loci predicted to be polymorphic by genome sequence analysis of three available Brucella genome sequences were tested for polymorphism by genotyping 2 I Brucella strains ( 18 reference strains representing the six 'classical' species and all biovars as well as 3 marine mammal strains currently recognized as members of two new species). The MLVA data efficiently cluster the strains as expected according to their species and biovar. For practical use, a subset of 15 loci preserving this clustering was selected and applied to the typing of 236 isolates. Using this MLVA-I5 assay, the clusters generated correspond to the classical biotyping scheme of Brucella spp. The 15 markers have been divided into two groups, one comprising 8 user-friendly minisatellite markers with a good species identification capability (panel I) and another complementary group of 7 microsatellite markers with higher discriminatory power (panel 2).

Conclusion: The MLVA-I 5 assay can be applied to large collections of Brucella strains with automated or manual procedures, and can be proposed as a complement, or even a substitute, of classical biotyping methods. This is facilitated by the fact that MLVA is based on non-infectious material (DNA) whereas the biotyping procedure itself requires the manipulation of the living agent. The data produced can be queried on a dedicated MLVA web service site.
\end{abstract}




\section{Background}

Brucellosis is a zoonosis affecting animals and humans worldwide. Brucella infections may result in significant economic losses due to abortion and slaughtering of infected animals. Humans are mainly infected through the consumption of contaminated dairy products or by direct contact with infected animals. In addition, certain Brucella spp have to be considered as potential biowarfare agents. Six species are currently recognized, B. abortus (8 biovars), B. melitensis (3 biovars), B. suis (5 biovars), $B$. ovis, B. canis and B. neotomae [1]. More recently, Brucella strains have been isolated from marine mammals [2], suggesting the existence of additional species $[3,4]$.

The genus Brucella is highly homogeneous (more than 90\% DNA/DNA homology [5]). Brucella classification is mainly based on differences in pathogenicity, host preferences, and conventional microbiological tests used for phenotyping (biotyping) [6]. Routine identification of Brucella species and biovars still relies on biotyping (reviewed in [7]). Only a few tools exist for further molecular subtyping, of which none has proven to be fully satisfactory for epidemiologic investigations or tracing back strains to their origin. Tandem repeat (TR) sequences may be an interesting class of markers, since multiple alleles can be present at a single locus, and size differences are easily resolved by electrophoresis (reviewed by $[8,9]$ ). Tandem repeats are often classified as microsatellites (repeat units up to $8 \mathrm{bp}$ ) and minisatellites [10,11]. Tandem repeat typing has proven to be highly appropriate for the typing of pathogenic bacterial species with a high genetic homogeneity, including the Mycobacterium tuberculosis complex, Bacillus anthracis, and Yersinia pestis [1215]. Recently, a family of tandem repeats located within a repeated sequence and present in multiple loci in the Brucella genome was used for strain typing $[16,17]$. The proposed set of eight microsatellite loci is extremely discriminant and highly efficient to distinguish strains within a local outbreak, but is unable to correctly predict the biovar or even the species of an isolate. A possible reason for that is the high mutation rate of these loci. Consequently, this MLVA assay cannot replace classical biotyping methods.

The availability of the whole genome sequences of $B$. melitensis $16 \mathrm{M}$, B. suis 1330 and B. abortus strain 9-941 [18-20] greatly facilitates the search for polymorphic DNA sequences [21]. In this report, we evaluated most tandem repeats showing at least two alleles among the three sequenced strains [22]. Eighteen reference strains and 3 strains isolated from marine mammals [23] were typed using these TR candidates to evaluate their associated polymorphism. For routine typing, a subset of 15 markers which enabled to cluster the isolates according to their biotype was selected. This set of markers was further eval- uated on a collection of 236 isolates representing the major biovars affecting terrestrial mammals (Table 1) to produce a first reference data set [see Additional file 1] which can be queried via the internet $[21,24]$.

\section{Results}

Evaluation of tandem repeats polymorphism

Comparison of the three genome sequences [21,22] identifies 107 TRs with a repeat unit larger than $5 \mathrm{bp}$ and predicted to display size polymorphism. Eighty of them were evaluated for polymorphism among 21 reference and marine mammal strains (Table 1). Twenty-two TRs (numbered Bruce 01 to Bruce 22 in Table 2 ) have three predicted alleles. Twelve of the 22 are octamers, five of which have been previously characterized [16].

Typing was done by PCR using the set of primers listed in Table 2, as described [13]. Six markers failed to amplify DNA satisfactorily, and were not included in the further study: they generated multiple band profiles (bruce20BRU329_8bp_148bp_7u; bruce38BRU1116_18bp_108bp_2u; bruce71BRU337_12bp_394bp_3u), or lacked amplification using the selected primers (bruce79BRU163_12bp_141bp_4u), or no appropriate primers could be designed targeting the flanking regions because of the presence of repeated elements (bruce76BRU243_21bp_2u; bruce77-BRU195_21bp_2u, not listed in Table 2).

Three markers (bruce44-BRU256_12bp_110bp_3u; bruce65-BRU824_41bp_182bp_2u; bruce69BRU488_57bp_181bp_1u) turned out to be monomorphic for the 21 reference strains. The results of the clustering analysis using the 71 remaining markers fits very well with the current knowledge of the degree of relationship between Brucella species [25] (Figure 1). We then looked for a subset of markers providing a similar discriminative power as the whole set for the collection of reference strains evaluated. Although extremely informative, the family of octamers, which includes the eight tandem repeats previously investigated $[16,17]$, are not appropriate for species/biovar discrimination because of their hypervariability and more stable markers must be used. Among the other markers, a set of the ten most polymorphic loci clusters the different species as expected. Two of these ten markers display allele size ranges not appropriate for analysis on currently available automated DNA fragments sizing machines such as capillary electrophoresis sequencing machines (Bruce 02 and Bruce 15 have alleles up to $2 \mathrm{~kb}$ and $5 \mathrm{~kb}$ respectively). The amplification patterns of the 21 reference strains using the other eight TRs are shown in Figure 2. These 8 markers (Bruce06, 08, $11,12,42,43,45,55)$ will subsequently be called MLVA typing panel 1 . These are minisatellites loci with repeat 
Table I: Brucella strains studied (reference and field strains)

\begin{tabular}{|c|c|c|c|}
\hline \multicolumn{4}{|c|}{ Reference and marine strains } \\
\hline Species & Biovar & Strain & Host \\
\hline B. abortus & 1 & 544 (ATCC 23448; BCCN R4)a & Cattle \\
\hline B. abortus & 2 & 86/8/59 (ATCC 23449; BCCN R5)a & Cattle \\
\hline B. abortus & 3 & Tulya (ATCC 23450; BCCN R6)a & Human \\
\hline B. abortus & 4 & 292 (ATCC 2345I; BCCN R7) & Cattle \\
\hline B. abortus & 5 & B3196 (ATCC 23452; BCCN R8) & Cattle \\
\hline B. abortus & 6 & 870 (ATCC 23453; BCCN R9) & Cattle \\
\hline B. abortus & 9 & C68 (ATCC 23455; BCCN RII)a & Cattle \\
\hline B. melitensis & I & I6 M (ATCC 23456; BCCN RI)a & Goat \\
\hline B. melitensis & 2 & 63/9 (ATCC 23457; BCCN R2)a & Goat \\
\hline B. melitensis & 3 & Ether (ATCC 23458; BCCN R3) & Goat \\
\hline B. suis & 1 & 1330 (ATCC 23444; BCCN R I2)a & Swine \\
\hline B. suis & 2 & Thomsen (ATCC 23445; BCCN RI3)a & Swine \\
\hline B. suis & 3 & $686($ ATCC 23446; BCCN R I4)a & Swine \\
\hline B. suis & 4 & $40($ ATCC 23447; BCCN RI5) & Reindeer \\
\hline B. suis & 5 & $513(\mathrm{BCCN} \mathrm{R21})^{\mathrm{a}}$ & Wild rodent \\
\hline B. ovis & & 63/290 (ATCC 25840; BCCN RI7)a & Sheep \\
\hline B. canis & & RM6/66 (ATCC 23365; BCCN RI8)a & Dog \\
\hline B. neotomae & & 5K33 (ATCC 23459; BCCN RI6) & Desert rat \\
\hline B. pinnipediae & & B2/94 (BCCN 94-73) & Common seal \\
\hline B. cetaceae & & $\mathrm{BI} / 94$ (BCCN 94-74) & Porpoise \\
\hline B. cetaceae & & BI4/94 (BCCN 94-75) & Common Dolphin \\
\hline \multicolumn{4}{|c|}{ Overview of the 236 additional isolates } \\
\hline Species & Biovar & Number of isolates investigated & \\
\hline B. abortus & 1 & 14 & \\
\hline B. abortus & 3 & 20 & \\
\hline B. abortus & 4 & 1 & \\
\hline B. abortus & 6 & 5 & \\
\hline B. abortus & 7 & 2 & \\
\hline B. abortus & 9 & 2 & \\
\hline B. abortus & (rough) & 1 & \\
\hline B. melitensis & I & 13 & \\
\hline B. melitensis & 2 & 13 & \\
\hline B. melitensis & 3 & 11 & \\
\hline B. melitensis & (atypical) & 1 & \\
\hline B. melitensis & (rough) & 2 & \\
\hline B. suis & 1 & 13 & \\
\hline B. suis & 2 & 87 & \\
\hline B. suis & 3 & 5 & \\
\hline B. suis & 4 & 5 & \\
\hline B. suis & 5 & 1 & \\
\hline B. suis & (rough) & 1 & \\
\hline B. ovis & & 23 & \\
\hline B. canis & & 16 & \\
\hline total & & 236 & \\
\hline
\end{tabular}

a: Reference strain

ATCC, American type culture collection

BCCN, Brucella culture collection, Nouzilly, France 
Table 2: List of tandem repeat loci investigated

22 tandem repeats with a predicted different length in the 3 genomes:

\begin{tabular}{|c|c|c|c|c|c|c|c|c|c|c|c|}
\hline vntra & alias name ${ }^{a}$ & $\mathrm{Chr}$ & $\%^{b}$ & upper primer & lower primer & b.suisc & b.melc & b.aborc & $\begin{array}{c}\text { nb of } \\
\text { different } \\
\text { alleles }^{d}\end{array}$ & $\min -\max b \mathbf{b}^{e}$ & HGDI $f$ \\
\hline BRUI938_8bp_37Ibp_9u & $\begin{array}{l}\text { Bruce01 or } \\
\text { TR7 }\end{array}$ & $\mathbf{I}$ & 100 & GGTCTGGGAAAACATGAAAAGC & AGCCGATCCTGCAAAACATAAT & 395 & 371 & 419 & 12 & $331-435$ & 0.95 \\
\hline BRUI923_339bp_787bp_3u & Bruce02 & I & 94 & AACGCAGCATCACCAATGT & CCCAGATGTTCGGCTATAGTATG & 448 & 787 & 2143 & 6 & 448-1974 & 0.8 \\
\hline BRU1627_9bp_199bp_3u & Bruce03 & I & 82 & GGCTATTATTTCACCGGCAAGA & TCTTGATTTCCTTGCGATACCA & 208 & 199 & 217 & 4 & $154-217$ & 0.48 \\
\hline BRUI543_8bp_152bp_2u & $\begin{array}{l}\text { Bruce } 04 \text { or } \\
\text { TR6*** }\end{array}$ & $\mathbf{I}$ & 100 & CTGACGAAGGGAAGGCAATAAG & CGATCTGGAGATTATCGGGAAG & 184 & 152 & 160 & 8 & $152-208$ & 0.87 \\
\hline BRUI365_8bp_185bp_3u & Bruce05 & I & 84 & AAGTATCAGGAAGGGCAGGTTC & GGGAGTAGGGGAATAAGGGAAT & 193 & 185 & 201 & 4 & $185-217$ & 0.59 \\
\hline BRUI322_134bp_408bp_3u & Bruce06* & I & 94 & ATGGGATGTGGTAGGGTAATCG & GCGTGACAATCGACTTTTTTGTC & 274 & 408 & 542 & 4 & $140-542$ & 0.73 \\
\hline BRUI 250_8bp_158bp_5u & Bruce 07** & I & 100 & GCTGACGGGGAAGAACATCTAT & ACCCTTTTTCAGTCAAGGCAAA & 166 & 158 & 150 & 5 & $150-190$ & 0.78 \\
\hline BRUII34_I8bp_348bp_4u & Bruce08* & I & 75 & ATTATTCGCAGGCTCGTGATTC & ACAGAAGGTTTTTCCAGCTCGTC & 330 & 348 & 366 & 4 & $312-366$ & 0.53 \\
\hline BRU588_8bp_156bp_7u & $\begin{array}{l}\text { Bruce09 or } \\
\text { TR8** }\end{array}$ & I & 94 & GCGGATTCGTTCTTCAGTTATC & GGGAGTATGTTTTGGTTGTACATAG & 140 & 156 & 124 & 8 & $124-244$ & 0.72 \\
\hline BRU221_19bp_127bp_2u & Bruce 10 & I & 90 & ATCAATTCGCGGATATTTCACT & AGTGCGTTTCATATGTTTGCTG & 146 & 127 & 165 & 2 & $127-146$ & 0.26 \\
\hline BRU2II_63bp_257bp_2u & Bruce II* & I & 80 & CTGTTGATCTGACCTTTGCAACC & CCAGACAACAACCTACGTCCTG & 509 & 257 & 383 & 6 & $257-698$ & 0.84 \\
\hline BRU73_15bp_392bp_13u & Bruce 12* & 2 & 58 & CGGTAAATCAATTGTCCCATGA & GCCCAAGTTCAACAGGAGTTTC & 345 & 392 & 375 & 7 & $302-452$ & 0.82 \\
\hline BRUI9_8bp_196bp_2u & Bruce 13 & 2 & 100 & CGAACGATAGACCAGAACATGC & TTGAAAGAATCAGATAAGATAAAGCA & 204 & 196 & 220 & 10 & $196-300$ & 0.85 \\
\hline BRUI8_8bp_102bp_7u & Bruce 14 & 2 & 100 & $\begin{array}{l}\text { TTGCTTTATCTTATCTGATTCTTTCA } \\
\text { A }\end{array}$ & GGTGTCGTTGGAGATAGAGGTC & 142 & 102 & 94 & 11 & $70-214$ & 0.93 \\
\hline BRUI II 2_264bp_346bp_lu & Bruce 15 & 2 & $8 \mathbf{I}$ & GCGGTGTTGTGTCTGTGGATA & GCCGTCAGTATCCACGTCATAG & 875 & 346 & 4112 & 7 & $346-2458$ & 0.83 \\
\hline BRU548_8bp_152bp_3u & Bruce 16** & 2 & 100 & ACGGGAGTTTTTGTTGCTCAAT & GGCCATGTTTCCGTTGATTTAT & 168 & 152 & 176 & 8 & $144-240$ & 0.85 \\
\hline BRU344_5bp_I I Obp_3u & Bruce 17 & 2 & 100 & TTTTCACAGGGCATGTTCTCAG & CGCGTTTCGATTGTGGAAAATA & 125 & 110 & 115 & 6 & $110-135$ & 0.79 \\
\hline BRU339_8bp_I46bp_5u & Bruce 1 8** & 2 & 87 & TATGTTAGGGCAATAGGGCAGT & GATGGTTGAGAGCATTGTGAAG & 138 & 146 & 154 & 6 & $130-170$ & 0.82 \\
\hline BRU324_6bp_163bp_18u & Brucel9 & 2 & 59 & GACGACCCGGACCATGTCT & ACTTCACCGTAACGTCGTGGAT & 169 & 163 & 184 & 7 & $76-190$ & 0.79 \\
\hline BRU329_8bp_148bp_7u & $\begin{array}{l}\text { Bruce } 20 \text { or } \\
\text { TR4 }\end{array}$ & 2 & 100 & AATACTGGGTCCAGTCCGATG & AGCGCAGCGACCATATTCT & 100 & 148 & 124 & ND & ND & ND \\
\hline BRU329_8bp_148bp_6u & Bruce 21** & 2 & 68 & CTCATGCGCAACCAAAACA & GATCTCGTGGTCGATAATCTCATT & 175 & 148 & 164 & 3 & 148-175 & 0.57 \\
\hline BRU322_8bp_158bp_6u & $\begin{array}{l}\text { Bruce } 22 \text { or } \\
\text { TRI }\end{array}$ & 2 & 98 & GATGAAGACGGCTATCGACTG & TAGGGGAGTATGTTTTGGTTGC & 150 & 158 & 134 & 10 & $125-2 \mid 4$ & 0.9 \\
\hline
\end{tabular}

56 tandem repeats with a predicted different length in $2 / 3$ genomes:

\begin{tabular}{|c|c|c|c|c|c|c|c|c|c|c|c|}
\hline vntra & alias name & Chr & $\%^{b}$ & upper primer & lower primer & b.suisc & b.melc & b.aborc & $\begin{array}{l}\text { nb of } \\
\text { different } \\
\text { alleles }^{\mathrm{e}}\end{array}$ & $\min -\max b p^{f}$ & HGDI $g$ \\
\hline BRUI990_9bp_I52bp_lu & Bruce23 & I & 88 & ATCAGCGAGTCGAAGGTCAGTT & TTCGACTATGCCAATCCAGATG & 161 & 152 & 161 & 2 & $|52-16|$ & 0.26 \\
\hline BRUI940_8bp_146bp_8u & $\begin{array}{l}\text { Bruce24 or } \\
\text { TR5 }\end{array}$ & 1 & 100 & AGGGGAGTATGTTTTTGGTTGC & GCTACAAGATCGAAGTGCTCCA & 146 & 146 & 106 & 10 & $106-217$ & 0.88 \\
\hline BRU1915_8bp_2I5bp_2u & $\begin{array}{l}\text { Bruce 25 or } \\
\text { TR3 }\end{array}$ & 1 & 100 & GGGAGTATGTTTTGGTTGCACA & CTATTTCGTCCTGCCATTCGAC & 239 & 215 & 239 & 8 & $215-736$ & 0.9 \\
\hline BRU1704_12bp_189bp_5u & Bruce26 & I & 68 & TCTTCATCCTGCGAGATCATGT & ATTCGTGATCGGGGTGATGAT & 162 & 189 & 189 & 2 & $162-189$ & 0.5 \\
\hline BRU1609_8bp_170bp_6u & Bruce27 & I & 76 & TCGACGTCGTCTGACATTTTCT & GGGAGTAAGGCAGTAGGGGAAT & 170 & 170 & 162 & 3 & $162-178$ & 0.61 \\
\hline BRU1599_IIbp_I47bp_4u & Bruce28 & I & 95 & TATCTTCCACGGCCATGAATC & GGCAGGATCGGCGTATAGATAA & 136 & 147 & 136 & 2 & $136-147$ & 0.1 \\
\hline BRUI528_15bp_132bp_3u & Bruce29 & 1 & 81 & TTGCGTTATTGATTGTCAGCAC & GCTGTGGCTCGTCTATGTGG & 132 & 132 & 117 & 2 & $117-132$ & 0.47 \\
\hline BRU I505_8bp_I5 Ibp_6u & $\begin{array}{l}\text { Bruce } 30 \text { or } \\
\text { TR2*** }\end{array}$ & 1 & 96 & TGACCGCAAAACCATATCCTTC & TATGTGCAGAGCTTCATGTTCG & 127 & 151 & 151 & 5 & || $9-|5|$ & 0.7 \\
\hline BRUI475_18bp_I20bp_lu & Bruce3I & 1 & 100 & GCTGAATCTTTTCCGCATCCT & GGAATCTCTGCACTGACAAAGC & 138 & 120 & 120 & 2 & $120-138$ & 0.51 \\
\hline
\end{tabular}


Table 2: List of tandem repeat loci investigated (Continued)

\begin{tabular}{|c|c|c|c|c|c|c|c|c|c|c|c|}
\hline BRU1424_8bp_106bp_5u & Bruce32 & $T$ & 88 & AGGTTTCCGGCGATAATGG & TCGGGATGCGCTCTAGAATATC & 106 & 106 & 98 & 3 & $98-114$ & 0.6 \\
\hline BRUI4I3_15bp_158bp_4u & Bruce33 & I & 81 & GATGGAGCTTGGTTCCTGCTT & GCATGATCCGTTTTCTTCTCAA & 143 & 158 & 143 & 3 & $128-158$ & 0.5 \\
\hline BRUI409_18bp_83bp_2u & Bruce34 & I & 84 & GCGATCGAAGGAAATATCGAG & CGCTGCCGGGATGTGAAC & 101 & 83 & 101 & 2 & $83-101$ & 0.26 \\
\hline BRUI 282_10bp_136bp_4u & Bruce35 & 1 & 91 & TGCGATAACAGGTGTACCCAAG & GACGGCAGCCATGCTGAT & 116 & 136 & 116 & 3 & $116-136$ & 0.27 \\
\hline BRUI234_15bp_157bp_4u & Bruce36 & 1 & 76 & TAAGGCTCTTGCGTTTGTATCG & TGCGTATCTTCAGACTGGCAAT & 142 & 157 & 142 & 2 & 142-157 & 0.38 \\
\hline BRUII76_2I bp_124bp_2u & Bruce37 & I & 95 & CCAAGCGTATCATCGATCTGTC & TCGGACGCAGATTGTTTCTATC & 103 & 124 & 123 & 2 & $103-124$ & 0.51 \\
\hline BRUIII6_18bp_108bp_2u & Bruce38 & 1 & 100 & CTGAATTGGGAGGAGGAACCAG & AGCTGAACGACCTTGGCATCT & 126 & 108 & 108 & 2 & $108-126$ & ND \\
\hline BRUIII2_15bp_164bp_7u & Bruce39 & 1 & 66 & GAAGGTCTCGAAGGAAGAGCTG & CCATCCATATTGATCGTCAGGA & 164 & 164 & 146 & 2 & $146-164$ & $0.5 \mathrm{I}$ \\
\hline BRU1048_15bp_94bp_2u & Bruce40 & I & 85 & AAAAGAAGGGTTTCCCCATACC & GGAAAGGACAGCTTCGAGTACC & 109 & 94 & 109 & 3 & $94-109$ & 0.34 \\
\hline BRUI1030_13bp_94bp_lu & Bruce4I & I & 100 & TTATGTCACCGCTGACGAATTT & CTCATTATGGACCCGGTCTTTC & 107 & 94 & 107 & 2 & 94-107 & 0.1 \\
\hline BRU424_125bp_539bp_4u & Bruce42* & 1 & 96 & CATCGCCTCAACTATACCGTCA & ACCGCAAAATTTACGCATCG & 538 & 539 & 289 & 5 & $164-789$ & 0.75 \\
\hline BRU379_12bp_182bp_2u & Bruce43* & I & 69 & TCTCAAGCCCGATATGGAGAAT & TATTTTCCGCCTGCCCATAAAC & 170 & 182 & 182 & 3 & 170-194 & 0.55 \\
\hline BRU256_12bp_I I0bp_3u & Bruce44 & I & 96 & GGCGCAAGATCGGAATGC & AGGCAGGTGCTGATTCTCCT & 110 & 110 & 122 & I & 110 & 0 \\
\hline BRU233_18bp_15 Ibp_3u & Bruce45* & 1 & 70 & ATCCTTGCCTCTCCCTACCAG & CGGGTAAATATCAATGGCTTGG & 187 & 151 & 151 & 4 & 133-187 & 0.65 \\
\hline BRU217_15bp_256bp_4u & Bruce46 & I & 81 & AAAAGCTTCCGAACCAAGTGTC & GGAGCTGGTTGAGCGTTATTTC & 256 & 256 & 241 & 2 & $24 I-256$ & 0.5 \\
\hline BRU149_15bp_II6bp_2u & Bruce47 & I & 90 & CTGCCAAGGGCGAGATAAAC & CATCGTTCTGATCTTCGTGACC & 131 & 116 & 131 & 2 & $|16-13|$ & 0.26 \\
\hline BRU|3|_29bp_13 Ibp_2u & Bruce48 & 1 & 95 & TATAAGTCCAGCCCATGACAGG & GCGGAATATCTGGATGGGATAC & $13 \mid$ & $|3|$ & 160 & 2 & $131-160$ & 0.51 \\
\hline BRUII2_13bp_266bp_2u & Bruce49 & I & 97 & AACCTCGGTCTATGATGCAACC & ACGCAGGGTTAGGTTTCTCAAA & 279 & 266 & 265 & 2 & 266-279 & 0.52 \\
\hline BRU80_1 2bp_162bp_3u & Bruce50 & I & 75 & GCAGAACCTGATGAACAACCTG & ATTTTCTGGTCGAGATCGAAGG & 174 & 162 & 174 & 2 & $162-174$ & 0.26 \\
\hline BRU80_15bp_74bp_2u & Bruce5I & 1 & 90 & TGACATGATGCAGAAAATGCAG & GTCCCTTGCCGCCTTTCAT & 74 & 74 & 89 & 2 & 74-89 & 0.47 \\
\hline BRU50_15bp_185bp_3u & Bruce52 & I & 95 & CAATGAACCAGATCAGCTTTCG & CGCCATGGTTTCAATATCACC & 170 & 185 & 170 & 2 & $170-185$ & 0.26 \\
\hline BRU50_30bp_ll Obp_lu & Bruce53 & I & 70 & CGGTTATGGTGTGGAGCAACT & CTTCCAGCGGGCTTTCAG & 140 & 110 & 110 & 2 & $110-140$ & 0.52 \\
\hline BRU28_13bp_150bp_lu & Bruce54 & 1 & 100 & CCGATCACAGACACAACAACTTC & GCGAAAAGGGAGCAGACATTAT & 163 & 150 & 163 & 2 & $150-163$ & 0.26 \\
\hline BRU2066_40bp_273bp_3u & Bruce55* & I & 80 & TCAGGCTGTTTCGTCATGTCTT & AATCTGGCGTTCGAGTTGTTCT & 234 & 273 & 273 & 5 & 194-354 & 0.69 \\
\hline BRU2028_| 2bp_135bp_4u & Bruce56 & I & 79 & TTGGTCGTTAGAACAAGAGTGG & CTGAACCTGTTCCGTCAAATCA & 135 & 135 & 123 & 2 & $123-135$ & 0.38 \\
\hline BRU69_9bp_30Ibp_4u & Bruce57 & 2 & 90 & ATGGGAGCCTATTTCGCTTACA & GGCGGTAGAATGGATAGCTCAC & 292 & 301 & 292 & 3 & 283-301 & 0.19 \\
\hline BRU33_24bp_98bp_lu & Bruce58 & 2 & 82 & CATCCTGCTTGGTGTTCTTTTTG & GATGGTCGTCACCAAGTCCAG & 122 & 98 & 122 & 2 & $98-122$ & 0.32 \\
\hline BRU33_9bp_256bp_7u & Bruce59 & 2 & 78 & CGTATCATCCGGCAATGGT & СTTTCTCTTTGTCGTGGGCTTC & 265 & 256 & 256 & 2 & 256-265 & 0.38 \\
\hline BRU24_12bp_279bp_3u & Bruce60 & 2 & 90 & AGCAAATGAATATGTCGCGTTG & TTCACCCCGATATCGATGAAT & 267 & 279 & 279 & 2 & 267-279 & 0.38 \\
\hline BRU22_1 2bp_162bp_5u & Bruce6I & 2 & 75 & CCTAATTTCGCCATTCGGTAAC & TTGCGGATTTTCCGAATAGAAC & 162 & 162 & 150 & 2 & $150-162$ & 0.5 \\
\hline BRU979_18bp_140bp_3u & Bruce62 & 2 & 85 & CTGACGCAGGGAAGCTTTGT & GAAAGAATGGTGAGCAGCAAGA & 155 & 140 & 155 & 2 & $140-155$ & 0.26 \\
\hline BRU833_15bp_145bp_3u & Bruce63 & 2 & 77 & AGGGTGACATTTGTTGGAGTCA & GTGGACAGACCCATGGTAAACG & 160 & 145 & 145 & 2 & $145-160$ & 0.51 \\
\hline BRU832_14bp_104bp_lu & Bruce64 & 2 & 93 & GAGACGACGCTTGAGGTTTTTC & CTTCCGGCGCTTCTTTCTTAT & 118 & 104 & 118 & 2 & $104-118$ & 0.26 \\
\hline BRU824_4l bp_182bp_2u & Bruce65 & 2 & 94 & CGCTCTAGGCATAGCTGTTGTG & GTCCAGCCGATAACCTTGCTAT & 143 & 182 & 143 & I & 142 & 0 \\
\hline BRU652_17bp_9lbp_lu & Bruce66 & 2 & 100 & ATATCCATGCGGGAAGGAAGAT & TATGCAGTGGGCATTCTCTACG & 108 & 91 & 91 & 2 & $91-108$ & 0.32 \\
\hline BRU609_3 Ibp_155bp_2u & Bruce67 & 2 & 68 & GCACCAGTGCGAGAAAATAGTG & AATTTTCCTTCGCGACTCCTTT & 124 & 155 & 155 & 2 & 124-155 & 0.52 \\
\hline BRU564_18bp_IIIbp_3u & Bruce68 & 2 & 79 & GACAACGATCCGCAGAAAGG & GTCAATGCCCTGATCGGTATC & 111 & III & 93 & 2 & $93-111$ & 0.47 \\
\hline BRU488_57bp_18I lbp_lu & Bruce69 & 2 & 100 & CGATGACAGAGCAAGACCGTTA & TTGACCGATAATTCTGCAATGG & 181 & 181 & 238 & I & 181 & 0 \\
\hline BRU339_2Ibp_146bp_2u & Bruce70 & 2 & 74 & GAGTAAGGCGAATAGGGGGAAC & AACTCTTCTTCCGCGACAACAC & 146 & 146 & 162 & 2 & $146-162$ & 0.51 \\
\hline BRU337_12bp_394bp_3u & Bruce7I & 2 & 79 & GAAGACGGCTATCGACTGGTCT & ACAAGCTCTATTCGCCTTACGC & 370 & 394 & 370 & ND & ND & ND \\
\hline BRU322_8bp_230bp_8u & Bruce72 & 2 & 66 & GAAGACGGCTATCGACTGGTCT & GTTTCAATGAAGGCGAGGTGAG & 206 & 230 & 206 & 10 & 198-294 & 0.87 \\
\hline BRU285_28bp_178bp_3u & Bruce73 & 2 & 86 & GTGGAAGGCGTTGTCATTCTG & ATCGGTCATGGTCTATCCTTCC & 178 & 178 & 150 & 2 & $150-178$ & 0.51 \\
\hline BRU275_8bp_147bp_6u & Bruce74 & 2 & 85 & GGATGAGGATTGAGGGCTTTT & ACGCAGACGATTTAACAAAGCA & 139 & 147 & 139 & 4 & $13 \mid-155$ & 0.63 \\
\hline BRU250_19bp_82bp_2u & Bruce75 & 2 & 100 & AGGACTATCAGGTGCGTGACAA & AAGGAAGACGTCGCTGAAAGAC & 82 & 82 & 101 & 2 & $82-10$ & 0.52 \\
\hline BRU|8I_14bp_122bp_2u & Bruce78 & 2 & 87 & CTAACAAATGACGGCAGAGTGC & TTGAACGCAAGCTTATCCAAAA & 136 & 122 & 136 & 2 & $122-136$ & 0.32 \\
\hline BRU|63_12bp_14lbp_4u & Bruce79 & 2 & 65 & TCCTGTTGAACGCAAGCTAATG & ATACTTCAGGCGGGGAGGAC & 153 & 141 & 153 & ND & ND & ND \\
\hline BRU542_12bp_178bp_4u & Bruce80 & 2 & 87 & CGAGGAATGTCAGGAAGATCAC & ACACAGACGCCAAAAGACAAA & 166 & 178 & 166 & 2 & $166-178$ & 0.18 \\
\hline
\end{tabular}

* markers constituting MLVA-I5 ; the 8 non-octamers $(*)$ are panel I, the 7 octamers $(* *)$ are panel 2

a naming nomenclature includes repeat unit size, PCR product size in strain I6M, corresponding repeat copy number, and common name;

b internal repeat homogeneity ; c expected PCR product size in each of the three sequenced genomes, Brucella suis I330, B. melitensis I6M, B. abortus 9-94I;

${ }^{d}$ number of alleles observed in the $2 \mathrm{I}$ strains ; e observed size range ; ${ }^{f} \mathrm{HGDI}$ : Hunter-Gaston diversity index ; ND, not determined 


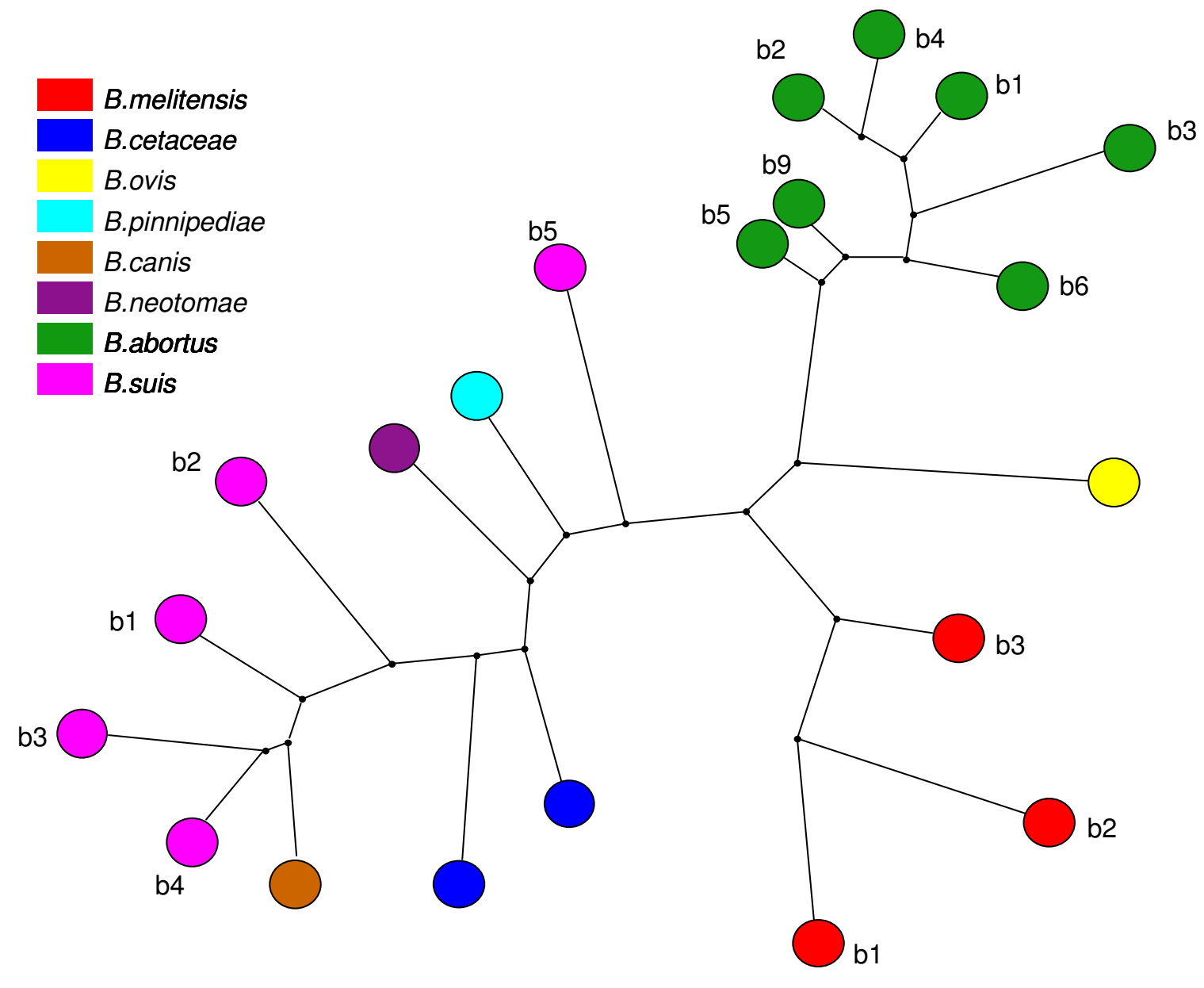

\section{Figure I}

Maximum parsimony analysis, on 2 I reference strains using data from all $7 \mathbf{l}$ markers. The different species are represented by different colours, as indicated. Biovars (b) are mentioned wherever relevant.

units length above 9 bp [10]. In addition, 7 robust and highly polymorphic octamers (microsatellites) were selected to constitute MLVA typing panel 2. Panel 2 comprises Bruce04 (designated as TR6 in [16]), Bruce07, Bruce09 (TR8), Bruce16, Bruce18, Bruce21 and Bruce30 (TR2).

\section{Evaluation of a MLVA assay comprising 15 markers}

The set of 15 TR markers (panel 1 and 2, listed with one or two asterisk in Table 2) was used for typing a larger collection of biotyped isolates including various species and biovars [see Additional file 1]. Among the 257 strains, panel 1 alone resolves 51 genotypes. This panel does not distinguish B. suis biovar 4 and B. canis. All B. canis strains investigated share panel 1 genotype 2 with some of the $B$. suis biovar 4 strains (Figure 3 ). Similarly, most B. suis biovar 3 strains share panel 1 genotype 4 with $B$. suis biovar
1. Panel 2 alone discriminates 200 genotypes. However, the resulting clustering only approximately fits with the expected species and biovar assignment. When using panel 1 and panel 2 together (MLVA-15 assay), 204 genotypes can be differentiated. The clustering analysis is shown in Figure 3, 4 and 5. A number of major clusters weakly connected to each other can be identified: B. suis biovar 1 (Figure 3), B. suis biovar 2( Figure 3 and figure 4), B. abortus (2 clusters, Figure 4 and Figure 5), B. melitensis (3 clusters, figure 5), B. ovis (Figure 3). Brucella suis biovar $5, B$. neotomae and the marine mammal strains are quite distinct from the closest strains (Figure 4). Brucella canis and $B$. suis biovar 4 are closely related and loosely connected to the $B$. suis biovar 1 cluster (Figure 3 ). The three $B$. melitensis clusters fit moderately with the biotyping results. Similarly, B. suis biovar 3 strains do not constitute a consistent group. 

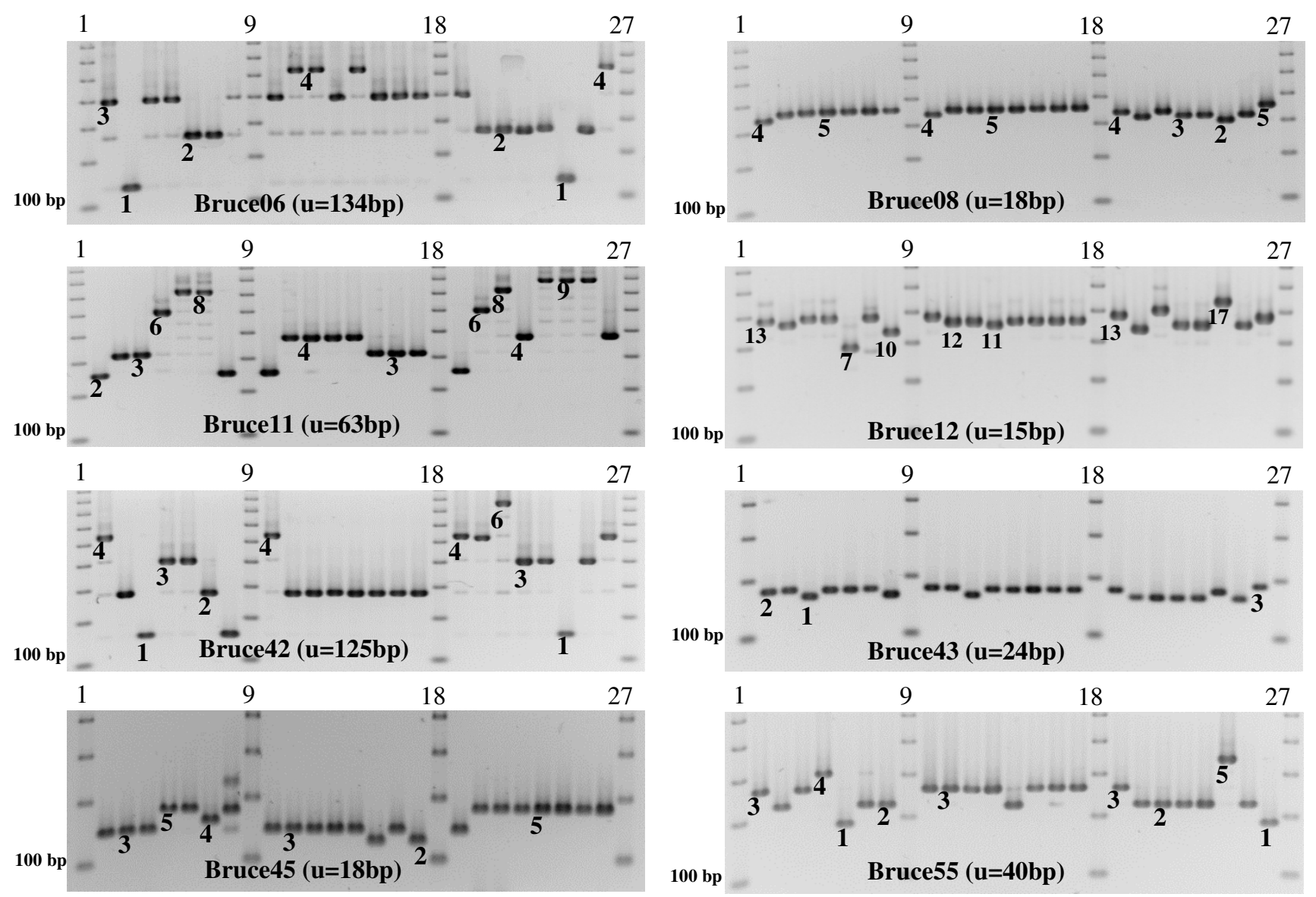

\section{Figure 2}

Amplification patterns of MLVA panel I on the 2 I reference strains. Lanes $2-8=2: B$. melitensis-bv I (I6M reference strain) ; 3: B. melitensis-bv2; 4: B. melitensis-bv3; 5: B. pinnipediae B2/94; 6: B. cetaceae BI/94; 7: B. cetaceae BI4/94; 8: B. ovis. Lanes I0-I7 = I0: B. melitensis-bvI (I6M) ; II: B. abortus-bvI; I2: B. abortus-bv2; I3: B. abortus-bv3; I4: B. abortus-bv4; I 5: B. abortus-bv5; 16: B. abortus-bv6; I 7: B. abortus-bv9. Lanes 19-26 = I9: B. melitensis-bv| (I6M); 20: B. suis-bvI; 2I: B. suis-bv2; 22: B. suis-bv3; 23: B. suis-bv4; 24: B. suis-bv5; 25: B. canis; 26: B. neotomae. Lanes I;9; I8;27 = I00bp DNA ladder. The values for strain I6M are deduced from Table 2 . The values for the other strains can be deduced from the I6M value, taking into account the indicated tandem repeat unit size. Examples are indicated, the full data can be deduced from the additional file [see Additional file I].I

\section{Discussion}

The genus Brucella has been divided into species and biovars for a long time, but this classification has been discussed controversially since DNA-DNA hybridization has been applied. The genus proved to be highly monomorphic with a level of relatedness among all species higher than $90 \%$ [5]. This homogeneity complicated the development of molecular assays able to efficiently recognise the species-specific entities. This finding led to the proposal of a monospecies genus, i.e. B. melitensis. The classical species would be considered as biovars only. However, most bacteriologists did not accept this concept which has recently been rejected by the subcommittee of taxonomy [26]. The purpose of the present study was firstly to investigate the polymorphism of tandem repeat loci predicted to be polymorphic by comparing the data of the three different Brucella strains already sequenced and secondly to evaluate to which extend tandem repeat typing and classical biotyping clustering fit together. We evaluated most of these loci with a repeat unit of $5 \mathrm{bp}$ or more.

Polymorphism has been confirmed at 71 loci. DNA was amplified at every locus from all 21 reference strains, including the 3 marine mammal strains (except for 


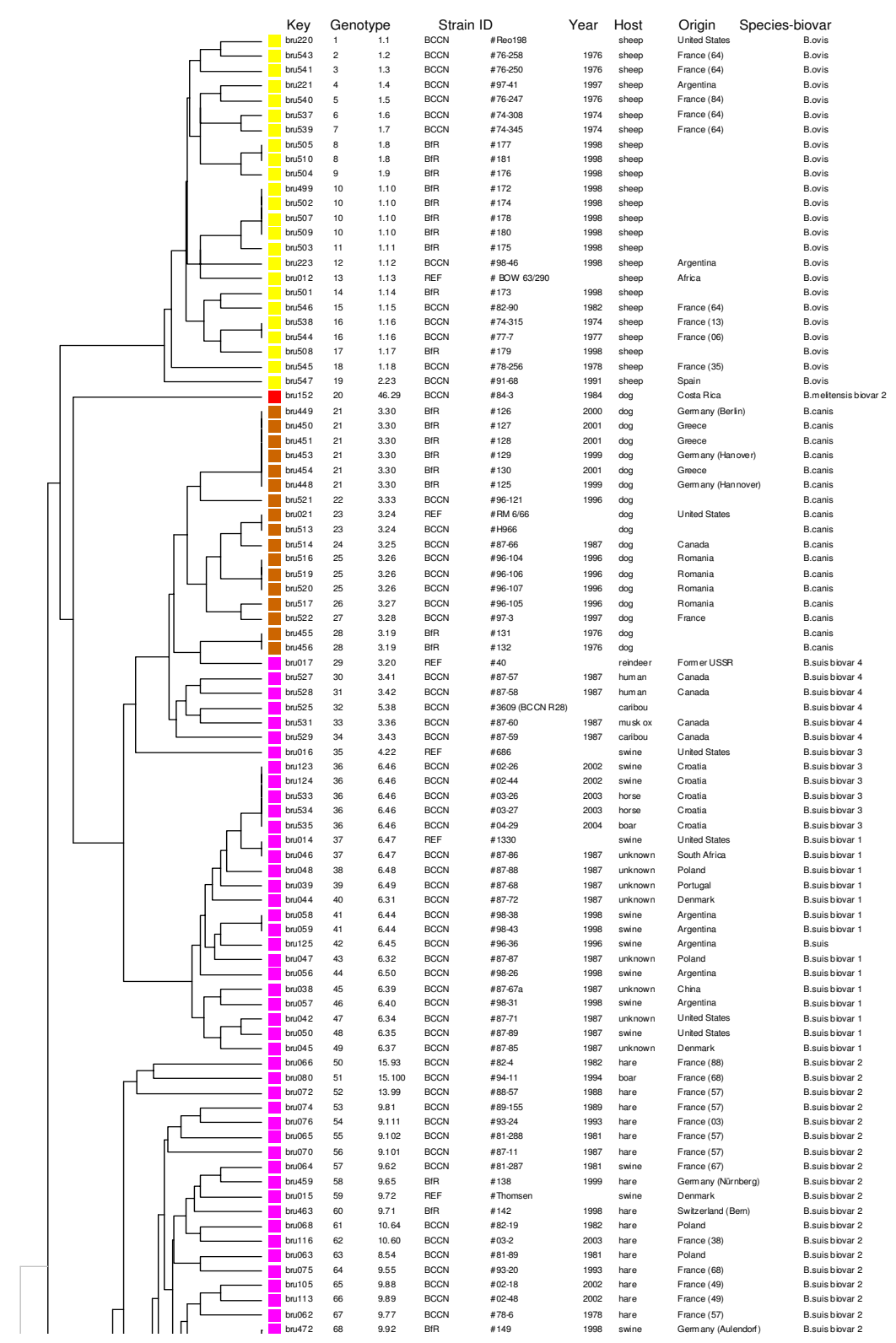

\section{Figure 3}

Clustering analysis in 257 strains and isolates with the two panels of markers (MLVA-I 5), genotypes I to 68. In the columns the following data are given from left to right : the DNA batch, the genotype, the strain ID including the name of the institution of origin ("BCCN" = Brucella Culture Collection from Nouzilly, INRA, Nouzilly, France ; "BfR" = Federal Institute for Risk Assessment, BfR, Berlin, Germany ; "REF" = the 21 reference and marine mammal strains, prepared by BCCN ; "vacc." = vaccine strain ), year of isolation, host and geographic origin when known, species and biovar (when relevant). The first genotype number (going from I to 204) is the MLVA-I5 genotype number. The second (for instance I.I) indicates the panel I genotype number (from I to 5I) followed by the panel 2 genotype number (from I to 200). The corresponding genotyping data can be found in the additional file [see Additional file I]. Wherever possible, the more precise geographic origin within a country is indicated (for instance France (03) is a strain originating from the French department number 03 (Allier) in the centre of France). The first part of the clustering of the 257 isolates in 204 genotypes is presented. It comprises 68 genotypes, corresponding to $B$. ovis, $B$. canis, $B$. suis biovar I, 3, 4, and part of the $B$. suis biovar 2 isolates. The colour code used is as shown in Figure I.I 


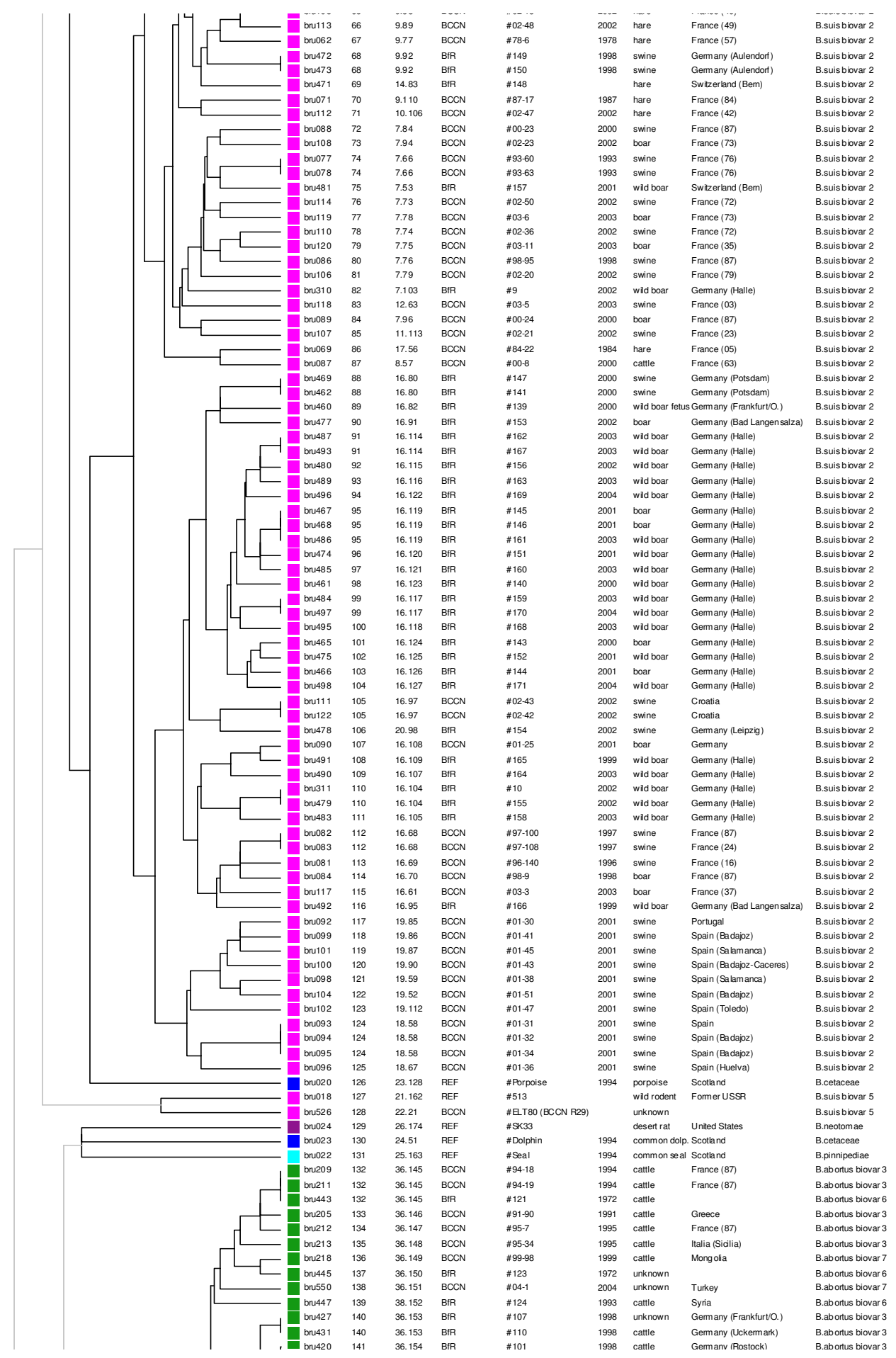

\section{Figure 4}

The columns content is as indicated in Figure 3 legend. The corresponding genotyping data can be found in the additional file [see Additional file I]. The second part of the clustering is displayed, genotypes 66 to I4I, comprising the rest of $B$. suis biovar 2 isolates, the $B$. neotomae strain, the three marine strains, the $2 B$. suis biovar 5 isolates, and part of the $B$. abortus isolates. The colour code used is as shown in Figure I 


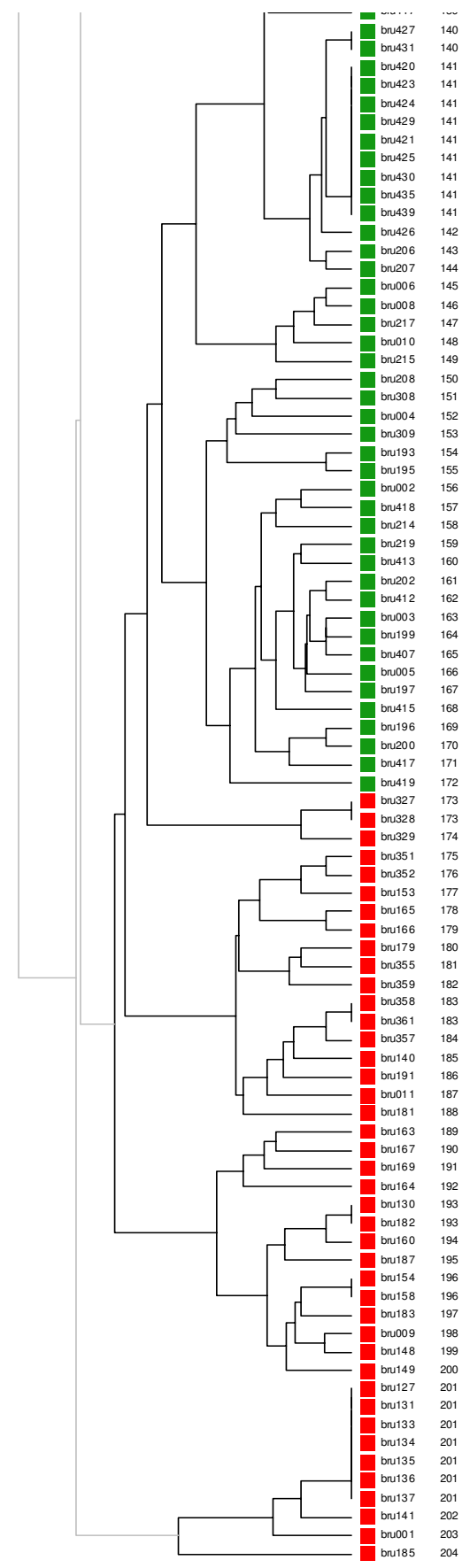

\begin{tabular}{|c|c|c|c|}
\hline$\ldots$ & 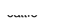 & 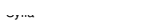 & \\
\hline 1998 & unknown & Gem any (Frankfurt/O.) & B.ab ortus biovar 3 \\
\hline 98 & cattle & Gem any (Uckem ark) & B.ab ortus biovar 3 \\
\hline 1998 & cattle & Germany (Rostock) & B.ab ortus biovar 3 \\
\hline 1998 & cattle & Gem any (Frankfurt/O.) & B.ab ortus biovar 3 \\
\hline 1998 & cattle & Germany (Frankfurt/O.) & B.ab ortus biovar 3 \\
\hline 1998 & cattle & Gemany (Frankfurt/O.) & B.ab ortus biovar 3 \\
\hline 1998 & cattle & Gemany (Frankfurt/O.) & B.ab ortus biovar 3 \\
\hline 1998 & cattle & Gemany (Frankfurto.) & B.ab ortus biovar 3 \\
\hline 1998 & cattle & Gemany (Uckemark) & B.ab ortus biovar 3 \\
\hline 1998 & cattle & Germany (Uckemmark) & B.ab ortus biovar 3 \\
\hline 1998 & cattle & Gemany (Uckemark) & B.ab ortus biovar 3 \\
\hline 1998 & cattle & Gemany (Frankfurt/O.) & B.ab ortus biovar 3 \\
\hline 1992 & cattle & France (85) & B.ab ortus biovar 3 \\
\hline \multirow{3}{*}{1993} & cattle & Spain (Pamplona) & B.ab ortus biovar 3 \\
\hline & cattle & England & B.ab ortus biovar 5 \\
\hline & cattle & England & B.ab ortus biovar 9 \\
\hline \multirow[t]{2}{*}{1987} & cattle & France (65) & B.ab ortus biovar 9 \\
\hline & cattle & Africa & B.ab ortus biovar 6 \\
\hline 1980 & dog & Belgium & B.ab ortus biovar 9 \\
\hline \multirow[t]{4}{*}{1993} & dromedary & Sudan & B.ab ortus biovar 3 \\
\hline & cattle & Thad & B.ab ortus biovar 6 \\
\hline & Human & Uganda & B.ab ortus biovar 3 \\
\hline & cattle & Tohad & B.ab ortus biovar 6 \\
\hline 1992 & cattle & France (87) & B.ab ortus biovar 1 \\
\hline \multirow[t]{2}{*}{1995} & cattle & France (63) & B.ab ortus biovar 1 \\
\hline & cattle & England & B.ab ortus biovar 1 \\
\hline 1972 & unknown & Switzerland (Bem) & B.ab ortus biovar 1 \\
\hline \multirow{6}{*}{1995} & cattle & Italia (Sidilia) & B.ab ortus biovar 4 \\
\hline & cattle & United States & B.abortus \\
\hline & cattle & Zimbabwe & B.ab ortus biovar 1 \\
\hline & cattle & United States & B.ab ortus biovar 1 \\
\hline & mouse & & B.ab ortus biovar 1 \\
\hline & cattle & England & B.ab ortus biovar 2 \\
\hline 1995 & cattle & Costa Rica & B.ab ortus biovar 1 \\
\hline 1996 & cattle & Switzerland (Bem) & B.ab ortus biovar 1 \\
\hline & cattle & England & B.ab ortus biovar 4 \\
\hline 1995 & cattle & Argentina & B.ab ortus biovar 1 \\
\hline & cattle & Zimbabwe & B.ab ortus biovar 1 \\
\hline 1995 & chamois & France (73) & B.ab ortus biovar 1 \\
\hline 1996 & cattle & Italia (Sidilia) & B.ab ortus biovar 1 \\
\hline 1972 & unknown & Germany (Lagesbüttel) & B.ab ortus biovar 1 \\
\hline 1972 & unknown & Germany (Müsterkap peln) & B.ab ortus biovar 1 \\
\hline & goat & United Arab Emirates & B.melitensis biovar 1 \\
\hline & goat & United Arab Emirates & B.melitensis biovar 1 \\
\hline & goat & United Arab Emirates & B.meitensis biovar 1 \\
\hline 1972 & unknown & & B.meitensis biovar 2 \\
\hline 1972 & unknown & & B.meitensis biovar 2 \\
\hline 1992 & sheep & Spain (Aragon) & B.meitensis biovar 2 \\
\hline 1996 & sheep & Israel & B.meitensis biovar 3 \\
\hline 1996 & sheep & Israel & B.mefitensis biovar 3 \\
\hline 1990 & cattle & Greece & B.melitensis biovar 3 \\
\hline 1999 & unknown & Gemany (Bonn) & B.meitensis biovar 2 \\
\hline 1990 & sheep & Syria (Aleppo) & B.meitensis biovar 2 \\
\hline 1991 & sheep & Syria & B.meitensis biovar 2 \\
\hline 1990 & sheep & Syria (Meskane) & B.melitensis biovar 2 \\
\hline 1993 & sheep & Syria & B.melitensis biovar 2 \\
\hline 1975 & unknown & Israel & B.meitensis biovar 1 \\
\hline 1994 & sheep & India & B.meltensis \\
\hline & goat & Turkey & B.melitensis biovar 2 \\
\hline 1992 & sheep & Spain (Basque Country) & B.mefitensis biovar 3 \\
\hline 1995 & sheep & Italia (Sidilia) & B.melitensis biovar 3 \\
\hline 1996 & sheep & Italia (Siölia) & B.meitensis biovar 3 \\
\hline 1996 & cattle & Italia (Sidilia) & B.melitensis biovar 3 \\
\hline 1995 & goat & Italia (Siodilia) & B.meitensis biovar 3 \\
\hline 1992 & unknown & Algeria & B.meitensis biovar 1 \\
\hline 1992 & unknown & Algeria & B.melitensis biovar 3 \\
\hline 1991 & sheep & Tunisia & B.meitensis biovar 3 \\
\hline 1992 & sheep & France (12) & B.melitensis \\
\hline 1995 & sheep & Italia (Siolilia) & B.meitensis biovar 2 \\
\hline 1996 & sheep & Italia (Siölia) & B.melitensis biovar 2 \\
\hline 1994 & cattle & France (12) & B.melitensis biovar 3 \\
\hline & goat & Italia & B.meitensis biovar 3 \\
\hline 1977 & goat & France $(06)$ & B.melitensis biovar 2 \\
\hline 1980 & sheep & France (04) & B.melitensis biovar 2 \\
\hline & goat & Mexico & B.melitensis biovar 1 \\
\hline 1996 & sheep & Israel & B.melitensis biovar 1 \\
\hline 1996 & unknown & Israel & B.mefitensis biovar 1 \\
\hline 1996 & sheep & Israel & B.melitensis biovar 1 \\
\hline & sheep & Israel & B.melitensis biovar 1 \\
\hline 96 & sheep & Israel & B.meltensis biovar 1 \\
\hline 96 & sheep & Israel & B.melitensis biovar 1 \\
\hline 1990 & unknown & South Africa & B.melitensis biovar 1 \\
\hline & goat & United States & B.melitensis biovar 1 \\
\hline & goat & Malta & B.melitensis \\
\hline
\end{tabular}

\section{Figure 5}

The columns content is as indicated in Figure 3 legend. The corresponding genotyping data can be found in the additional file [see Additional file I]. The third part of the clustering (genotypes I40 to 204) is displayed, comprising the rest of $B$. abortus isolates, and the B. melitensis isolates. The RB5I vaccine strain tested here is genotype 159, S19 is genotype 16I, and a number of Revl isolates share genotype 20I. The colour code used is as shown in Figure I. 
Bruce 04 in the B. melitensis bv 3 reference strain Ether and Bruce01 in the B.ovis reference strain BOW63/290) confirming the very high genetic homogeneity of the genus Brucella.

A MLVA typing assay depends on the selection of markers which individually would not provide a relevant clustering. Taken separately, the TR markers are either not informative enough, or too variable or show a high level of homoplasy. However, the combination of well selected independent loci may be highly discriminatory and to some extend phylogenetically relevant, as shown previously for other species [9], and demonstrated here for Brucella. We propose a selection of 15 markers to be used in a Brucella MLVA assay consisting of two complementary panels, panel 1 ( 8 markers) and panel 2 (7 markers). The fifteen markers are a combination of moderately variable (minisatellites, panel 1) and highly discriminant (microsatellites, panel 2) loci (Table 2).

The strain clustering achieved is consistent with wellestablished phenotypic and molecular characteristics (Figure 3, 4 and 5). The biovars 1, 2 and 4 of B. abortus are gathered in agreement with (i) the sensitivity to thionin and (ii) the PCR-RFLP pattern of the omp $2 a$ genes specific for these biovars [27]. B. abortus biovar 3 strains are found in a separate group except for 2 strains originated from Africa (BCCN 93-26 and the reference strain Tulya). Strains isolated in Africa often show distinct phenotypes [28] and thus, it is not surprising to find these two strains separated. The two strains do not require $\mathrm{CO}_{2}$ for growing. Their MLVA closest neighbours are two B. abortus biovar 6 strains also isolated in Africa. Assignment to biovar 3 or 6 reflects the $\mathrm{H}_{2} \mathrm{~S}$ production which is the unique phenotypical criteria to differentiate these two biovars. The MLVA assay confirms that some African strains significantly differ from isolates of other origin and that $B$. abortus biovar 3 is a heterogeneous group.

The B. melitensis group is very heterogeneous using either panel 1 or both panels (MLVA-15), and comprises four main subgroups. Biovar 2 and 3 strains are mixed in two groups, together with a few biovar 1 strains. The other biovar 1 isolates form 2 groups, one including the $16 \mathrm{M}$ reference strain, and the other (genotypes 173 and 174, Figure 5 ) comprising 3 isolates from the United Arab Emirates. $B$. melitensis BCCN 84-3 strain (MLVA-15 genotype 20) is an isolate from a dog in Costa Rica, which was biotyped as $B$. melitensis biovar 2, but appears to be distantly related to other B. melitensis strains. This strain is smooth as observed by the agglutination with anti-A serum, and the profile obtained in oxidative metabolism is typical of $B$. melitensis. Panel 1 analysis (not shown) does associate this strain with $B$. melitensis, but the full MLVA-15 analysis suggests a position closer to the B. canis group (Figure 3 ).
B. suis strains are clearly differentiated in three groups (Figures 3 and 4). A first group includes all biovar 1, 3, and 4 strains, and a second group all biovar 2 strains. The two rare biovar 5 strains are very distantly related. The correlation with biovars is good with some interesting exceptions. The five B. suis biovar 3 isolates from Croatia have the same genotype (MLVA-15 genotype 36, Figure 3 [see Additional file 1]), and cluster with $B$. suis biovar 1 strains but not with the reference $B$. suis biovar 3 strain. More $B$. suis strains phenotypically identified as biovar 3 from other geographic origins are required. This may suggest that the biovar 3 phenotype may have appeared independently more than once. Biovar 1 and biovar 3 strains are distinguished by sensitivity to fuchsine and ability to produce $\mathrm{H}_{2} \mathrm{~S}$. Atypical fuchsine-resistant biovar 1 strains have already been described [6], as well as atypical fuchsine-sensitive B. melitensis strains [29,30]. So both the fuchsine sensitivity, and the $\mathrm{H}_{2} \mathrm{~S}$ production (as suggested above for $B$. abortus) may appear to be phylogenetically weak markers with some degree of homoplasy. Among biovar 2, strains isolated from Spain and Portugal are related and can be distinguished from other European strains investigated. Biovar 4 strains can be found right beside B. canis. Meyer [31] has previously proposed a model for evolutionary derivation of Brucella organisms on the basis of phenotypic characteristics and proposed a close relationship between $B$. suis biovar $3 / 4$, and $B$. canis. PCR-RFLP analyses of the porin genes are in agreement with this finding [27].

Three classical vaccine strains were included, Rev.1 (genotype 201), S19 (genotype 161) and RB51 (genotype 159). Six other isolates, from Israel, share genotype 201. These streptomycin resistant isolates were confirmed as Rev.1 vaccine strains using the previously described assay [32] (data not shown). This is not unexpected since vaccination is used in this country, and simply illustrates the stability of the MLVA assay in the present case.

Strains clustering together frequently have a close or identical geographic origin, e.g. MLVA-15 genotype 16 comprises $2 \mathrm{~B}$. ovis isolates, coming from the same region of France "Provence-Côte d'Azur" (departments 06 and 13). In almost all such instances where the MLVA genotype of two isolates is identical, the available epidemiological data is indeed compatible with a common source of infection. The rare exceptions would then suggest that some strains travel efficiently. MLVA-15 genotype 132 was observed in Germany in 1972 and in the centre of France (department 87) in 1994. MLVA-15 genotype 1 (B. canis) was observed in Greece and Germany. More epidemiological data will be needed in order to draw precise conclusions on the circulation of the strains. 
The MLVA-15 results support the current classification of the genus Brucella. In addition, differences found by phenotypic identification and/or by molecular studies are also detected by MLVA. One major advantage of MLVA is the ease of data exchanges. The data itself can be summarized by a very simple flat text file containing the repeat copy numbers for each locus and each strain. This data can also be made accessible and queried across the internet as shown $[21,24]$.

Another advantage is that MLVA typing only depends on the measurement of DNA amplicon sizes, so that a number of electrophoretic techniques can be used, ranging from manual, low-cost, agarose gels, to high-throughput capillary electrophoresis sequencing machines.

In the near future, it is tempting to speculate that international databases containing MLVA data of thousands of strains will be produced, and MLVA will become a routine assay for any new isolate. We believe that the MLVA-15 assay will be one step in this direction. A first use of the assay for a clinical application was recently described [33].

\section{Methods \\ Bacterial strains}

The 257 strains and isolates used for MLVA typing are listed or described globally in Table 1. One hundred and seventeen B. suis, 43 B. melitensis, 52 B. abortus, 24 B. ovis, one B. neotomae, 17 B. canis and 3 strains isolated from marine mammals [2] were investigated. This collection includes the 18 classical reference strains representing the different species and biovars of Brucella. All strains were mainly isolated from animals and in a few cases from humans or unknown species (Figure 3,4 and 5), and were identified by phenotypical tests based on agglutination with monospecific antisera (serotyping), phage typing, dye sensitivity, $\mathrm{CO}_{2}$ requirement and $\mathrm{H}_{2} \mathrm{~S}$ production [6].

\section{Identification of variable number tandem repeats by genomic sequence comparison}

The methods previously described $[10,12,21,22]$ and the genome sequence data for $B$. suis strain $1330, B$. melitensis strain $16 \mathrm{M}$ and $B$. abortus strain 9-941 [18-20] were used to identify TRs that may help to differentiate closely related genomes.

The different TRs are designated by using the nomenclature previously described [13]. For instance BRU211_63bp_257bp_2u (bruce11) is a TR at position $211 \mathrm{~kb}$ in the B. melitensis $16 \mathrm{M}$ genome. Its common laboratory name (alias name) is Bruce 11 . It has a $63 \mathrm{bp}$ motif, and a total PCR product length of $257 \mathrm{bp}$ in the $B$. melitensis $16 \mathrm{M}$ strain when using the primer set indicated in Table 2. This allele size corresponds to 2 units.

\section{PCR amplification and genotyping}

Brucella DNA was prepared as previously described [27]. PCR amplification was performed in a total volume of 15 $\mu \mathrm{l}$ containing $1 \mathrm{ng}$ of DNA, $1 \times$ PCR Reaction Buffer, $1 \mathrm{U}$ of Taq DNA polymerase (Qbiogen, Illkirch, France), $200 \mu \mathrm{M}$ of each deoxynucleotide triphosphate, and $0.3 \mu \mathrm{M}$ of each flanking primer as described previously [15].

Amplifications were performed in a MJ Research PTC200 thermocycler. An initial denaturation step at $96^{\circ} \mathrm{C}$ for 5 minutes was followed by 30 cycles of denaturation at $96^{\circ} \mathrm{C}$ for $30 \mathrm{~s}$, primer annealing at $60^{\circ} \mathrm{C}$ for $30 \mathrm{~s}$, and elongation at $70^{\circ} \mathrm{C}$ for $1 \mathrm{~min}$. The final extension step was performed at $70^{\circ} \mathrm{C}$ for $5 \mathrm{~min}$.

Two to five microliters of the amplification product were loaded on a 3\% standard agarose gel for analyzing tandem repeats with a unit length shorter than $10 \mathrm{bp}$ and on a $2 \%$ standard agarose gel for all others, and run under a voltage of $8 \mathrm{~V} / \mathrm{cm}$ until the bromophenol blue dye had reached the $20 \mathrm{~cm}$ position. Gels were stained with ethidium bromide, visualized under UV light, and photographed (Vilber Lourmat, Marnes-la-Vallée, France). A 100-bp and a 20-bp ladder (EZ Load 100 pb or 20 bp PCR Molecular Ruler, Biorad, Marnes-la-Coquette, France) were used as molecular size markers depending on the tandem repeat unit length. Gel images were managed using the Bionumerics software package (version 4.0, Applied-Maths, Belgium).

\section{Data analysis}

Band size estimates were converted to a number of units within a character dataset using Bionumerics version 4.0 (Applied-Maths, Belgium) [see Additional file 1]. Clustering analyses used the categorical coefficient and UPGMA (unweighted pair group method using arithmetic averages). The use of the categorical parameter implies that the character states are considered unordered. The same weight is given to a large or a small number of differences in the number of repeats at each locus. Maximum parsimony was done using Bionumerics, running 200 bootstrap simulations and treating the data as categorical.

\section{Polymorphism index}

The Hunter Gaston diversity index [34] (HGDI) was used.

\section{Authors' contributions}

MG, IJ, SAD, KN, HN were in charge of strain selection, collection and checking of related data, preparation and provision of DNAs. PLF did the MLVA genotyping work. GV was in charge of the Bionumerics database, error checking, clustering analyses. FD and PB did the genome sequence analyses for polymorphic tandem repeat searches and the genotyping page. GV wrote the report. IJ 
and MG helped to draft the manuscript. All authors read, commented and approved the final manuscript.

\section{Additional material}

\section{Additional File 1}

MLVA-15 data for each of the 204 genotypes. The first three columns from the left are genotype numbers obtained with the different panels. The subsequent columns are the typing data itself. The first 8 markers (headings, bruce06 to bruce55) constitute panel 1 (minisatellites, tandem repeat unit length above $9 \mathrm{bp}$ ). The last 7 columns (starting from bruce04) constitute panel 2 (microsatellites, tandem repeat unit length up to 8 base-pairs).

Click here for file

[http://www.biomedcentral.com/content/supplementary/14712180-6-9-S1.txt]

\section{Acknowledgements}

We thank Drs A.P. MacMillan (LVA, Weybridge, UK) and B. Garin-Bastuji (AFSSA, Maisons-Alfort, France) for providing some of the strains used in this study. We thank Cornelia Goellner, Angelika Draeger and Csilla Lodri for their help to prepare the DNA and also for performing some identification work. Dr. Beatriz Guerra critically reviewed the manuscript. We thank Ibtissem Grissa for her contribution to the setting up of the Brucella genotyping page. Work on the typing of dangerous pathogens and reference databases and collections is supported by DGA grants (Délégation Générale pour l'Armement, PEA02-36-0I). This project is part of European biodefence project CEPAI 3.14 coordinating work on dangerous pathogens accountability from Sweden, Germany, France, Italy, the Nederland, and Norway, and of COST Action 845 (Brucellosis in animals and man).

\section{References}

I. Corbel MJ, Brinley Morgan W]: Genus Brucella Meyer and Shaw 1920, I73AL. In Bergey's Manual of Systematic Bacteriology Volume I. Edited by: Krieg NR, Holt JG. Baltimore: Williams and Wilkins; 1984:377-390.

2. Ross HM, Foster G, Reid RJ, Jahans KL, MacMillan AP: Brucella species infection in sea-mammals. Vet Rec 1994, 134:359.

3. Cloeckaert A, Verger JM, Grayon M, Paquet JY, Garin-Bastuji B, Foster $\mathrm{G}$, Godfroid J: Classification of Brucella spp. isolated from marine mammals by DNA polymorphism at the omp2 locus. Microbes Infect 200I, 3:729-738.

4. Cloeckaert A, Grayon M, Grepinet O, Boumedine KS: Classification of Brucella strains isolated from marine mammals by infrequent restriction site-PCR and development of specific PCR identification tests. Microbes Infect 2003, 5:593-602.

5. Verger JM, Grimont F, Grimont PAD, Grayon M: Brucella, a monospecific genus as shown by deoxyribonucleic acid hybridization. Int J Syst Bacteriol 1985, 35:292-295.

6. Alton GG, Jones LM, Angus RD, Verger JM: Techniques for the brucellosis laboratory Paris, France: INRA; 1988.

7. Cutler SJ, Whatmore AM, Commander NJ: Brucellosis - new aspects of an old disease. J Appl Microbiol 2005, 98: 1270-128I.

8. Lindstedt BA: Multiple-locus variable number tandem repeats analysis for genetic fingerprinting of pathogenic bacteria. Electrophoresis 2005, 26:2567-2582.

9. Vergnaud G, Pourcel C: Multiple Locus VNTR (Variable Number of Tandem Repeat) Analysis (MLVA). In Molecular Identification, Systematics and Population Structure of Prokaryotes Edited by: Stackebrandt E. Springer-Verlag; 2006:83-104.

10. Vergnaud G, Denoeud F: Minisatellites: Mutability and Genome Architecture. Genome Res 2000, 10:899-907.

II. Denoeud F, Vergnaud G, Benson G: Predicting Human Minisatellite Polymorphism. Genome Res 2003, 13:856-867.
12. Le Flèche P, Hauck Y, Onteniente L, Prieur A, Denoeud F, Ramisse V, Sylvestre P, Benson G, Ramisse F, Vergnaud G: A tandem repeats database for bacterial genomes: application to the genotyping of Yersinia pestis and Bacillus anthracis. BMC Microbiol 200I, I:2.

13. Le Flèche P, Fabre M, Denoeud F, Koeck JL, Vergnaud G: High resolution, on-line identification of strains from the Mycobacterium tuberculosis complex based on tandem repeat typing. BMC Microbiol 2002, 2:37.

14. Fabre M, Koeck JL, Le Fleche P, Simon F, Herve V, Vergnaud G, Pourcel $C$ : High genetic diversity revealed by variable-number tandem repeat genotyping and analysis of hsp65 gene polymorphism in a large collection of "Mycobacterium canettii " strains indicates that the $M$. tuberculosis complex is a recently emerged clone of "M. canettii". J Clin Microbiol 2004, 42:3248-3255.

15. Pourcel C, Andre-Mazeaud F, Neubauer H, Ramisse F, Vergnaud G: Tandem repeats analysis for the high resolution phylogenetic analysis of Yersinia pestis. BMC Microbiol 2004, 4:22.

16. Bricker BJ, Ewalt DR, Halling SM: Brucella 'Hoof-Prints': strain typing by multi-locus analysis of variable number tandem repeats (VNTRs). BMC Microbiol 2003, 3:15.

17. Bricker B], Ewalt DR: Evaluation of the HOOF-Print assay for typing Brucella abortus strains isolated from cattle in the United States: results with four performance criteria. $B M C$ Microbiol 2005, 5:37.

18. Paulsen IT, Seshadri R, Nelson KE, Eisen JA, Heidelberg JF, Read TD, Dodson RJ, Umayam L, Brinkac LM, Beanan MJ, Daugherty SC, Deboy RT, Durkin AS, Kolonay JF, Madupu R, Nelson WC, Ayodeji B, Kraul M, Shetty J, Malek J, Van Aken SE, Riedmuller S, Tettelin H, Gill SR, White O, Salzberg SL, Hoover DL, Lindler LE, Halling SM, Boyle SM, Fraser CM: The Brucella suis genome reveals fundamental similarities between animal and plant pathogens and symbionts. Proc Natl Acad Sci USA 2002, 99:13148-13153.

19. DelVecchio VG, Kapatral V, Redkar RJ, Patra G, Mujer C, Los T, Ivanova $N$, Anderson I, Bhattacharyya A, Lykidis A, Reznik G, Jablonski L, Larsen N, D'Souza M, Bernal A, Mazur M, Goltsman E, Selkov E, Elzer PH, Hagius S, O'Callaghan D, Letesson JJ, Haselkorn R, Kyrpides $\mathrm{N}$, Overbeek $\mathrm{R}$ : The genome sequence of the facultative intracellular pathogen Brucella melitensis. Proc Natl Acad Sci USA 2002, 99:443-448.

20. Halling SM, Peterson-Burch BD, Bricker BJ, Zuerner RL, Qing Z, Li LL, Kapur V, Alt DP, Olsen SC: Completion of the genome sequence of Brucella abortus and comparison to the highly similar genomes of Brucella melitensis and Brucella suis. J Bacteriol 2005, 187:2715-2726.

21. Denoeud F, Vergnaud G: Identification of polymorphic tandem repeats by direct comparison of genome sequence from different bacterial strains: a Web-based ressource. BMC Bioinformatics 2004, 5:4.

22. The Microorganisms Tandem Repeats Database [http://min isatellites.u-psud.fr]

23. Verger JM, Grayon M, Cloeckaert A, Lefevre M, Ageron E, Grimont $F$ : Classification of Brucella strains isolated from marine mammals using DNA-DNA hybridization and ribotyping. Res Microbiol 2000, I 5 I:797-799.

24. The MLVA Web Service [http://bacterial-genotyping.igmors.upsud.fr]

25. Moreno E, Cloeckaert A, Moriyon I: Brucella evolution and taxonomy. Vet Microbiol 2002, 90:209-227.

26. International Committee on Systematics of Prokaryotes (ICSP) [http://www.the-icsp.org/]

27. Cloeckaert A, Verger JM, Grayon M, Grepinet O: Restriction site polymorphism of the genes encoding the major $25 \mathrm{kDa}$ and $36 \mathrm{kDa}$ outer-membrane proteins of Brucella. Microbiology 1995, I4I:2III-2I21.

28. Verger JM, Grayon M: Characteristics of $\mathbf{2 7 3}$ strains of Brucella abortus of African origin. Dev Biol Stand 1984, 56:63-7I.

29. Banai M, Mayer I, Cohen A: Isolation, identification, and characterization in Israel of Brucella melitensis biovar I atypical strains susceptible to dyes and penicillin, indicating the evolution of a new variant. / Clin Microbiol 1990, 28: 1057-1059.

30. Corbel MJ: Identification of dye-sensitive strains of Brucella melitensis. J Clin Microbiol 1991, 29:1066-1068. 
31. Meyer ME: Current concepts in the taxonomy of the genus Brucella. In Animal brucellosis Edited by: Nielsen K, Duncan JR. Boca Raton, Floride: CRC Press; 1990:I-17.

32. Cloeckaert A, Grayon M, Grepinet O: Identification of Brucella melitensis vaccine strain Rev.I by PCR-RFLP based on a mutation in the rpsL gene. Vaccine 2002, 20:2546-2550.

33. Al Dahouk S, Hagen RM, Nockler K, Tomaso H, Wittig M, Scholz HC, Vergnaud G, Neubauer $\mathrm{H}$ : Failure of a short-term antibiotic therapy for human brucellosis using ciprofloxacin. A study on in vitro susceptibility of Brucella strains. Chemotherapy 2005, 5I:352-356.

34. Hunter PR, Gaston MA: Numerical index of the discriminatory ability of typing systems: an application of Simpson's index of diversity. J Clin Microbiol 1988, 26:2465-2466.

Publish with Bio Med Central and every scientist can read your work free of charge

"BioMed Central will be the most significant development for disseminating the results of biomedical research in our lifetime. "

Sir Paul Nurse, Cancer Research UK

Your research papers will be:

- available free of charge to the entire biomedical community

- peer reviewed and published immediately upon acceptance

- cited in PubMed and archived on PubMed Central

- yours - you keep the copyright

Submit your manuscript here:

http://www.biomedcentral.com/info/publishing_adv.asp
BioMedcentral 